\title{
Energy Efficiency of Large-Scale Wireless Networks: Proactive Versus Reactive Networking
}

\author{
Qing Zhao, Member, IEEE, and Lang Tong, Fellow, IEEE
}

\begin{abstract}
An analytical approach to the characterization of energy consumption of large-scale wireless networks is presented. The radio model includes energy consumption of nodes at various operating states. We analyze the total energy consumption of the proactive and the reactive networking strategies, taking into account transmitting, listening, and sleeping energy. Scaling laws with respect to the increase of node density and geographical size are derived. Energy efficiency and overhead at the physical and the network layers are evaluated against message duty cycle, channel fading rate, and node mobility. The crossover point in message duty cycle below which reactive network has assured advantages is obtained. The analysis is then applied to large-scale sensor networks for applications involving data-centric and location-centric queries. The ad hoc sensor network architecture is compared with sensor networks with mobile access points.
\end{abstract}

Index Terms-Energy consumption analysis, energy scaling law, mobile ad hoc networks, proactive and reactive networking, sensor networks.

\section{INTRODUCTION}

$\mathbf{M}$ OBILE ad hoc networks and sensor networks are crucial in network centric warfare. The mobility of nodes, the energy constraints, and the size of the network all impose formidable design challenges. Given the wide range of design options, there is a need for a fundamental understanding among various tradeoffs.

In this paper, we take a theoretical approach to analyzing energy consumption of general wireless ad hoc networks in which links are subject to block fading and network topology varies in time. We consider two design paradigms: proactive networking and reactive networking. By proactive networking we mean that all links between nodes and all routes between source-destination pairs are maintained and updated regardless of the data traffic. When a message arrives, it travels through a predetermined route to its destination. The reactive networking, in contrast, assumes no predetermined routes, nor does it maintain links at the physical layer. It finds a route only when a message is to be delivered from source to destination and establishes a link just before the transmission is to be scheduled.

Manuscript received April 29, 2004; revised January 19, 2005. This work was supported in part by the Multidisciplinary University Research Initiative (MURI) under the Office of Naval Research Contract N00014-00-1-0564, and in part by the Army Research Laboratory CTA on Communication and Networks under Grant DAAD19-01-2-0011.

Q. Zhao is with the Electrical and Computer Engineering, University of California, Davis, CA 95616 USA (e-mail: qzhao@ece.ucdavis.edu).

L. Tong is with the Electrical and Computer Engineering, Cornell University, Ithaca, NY 14853 USA (e-mail: 1tong @ ece.cornell.edu).

Digital Object Identifier 10.1109/JSAC.2005.845411
It is intuitive that reactive networking would be energy efficient when the traffic is light and inefficient when the traffic is heavy. A similar tradeoff can be made between a network with high mobility nodes and one with mostly stationary nodes. It is, however, far from obvious how the energy consumption scales with the number of the nodes in the network, by increasing either the density of the nodes or the physical size of the network. One may also want to know which part of the network operation is the most energy consuming or the least energy efficient. Is the energy spent at the physical layer or at the network layer the dominating factor? Is the transmitting or the listening the primary energy drain? Such questions, while possible to be tackled via simulations, are more suitable for an analytical approach.

\section{A. Summary of Results and Organization}

The network, channel, and radio models are specified in Section II. In Section III, we present the point-to-point traffic analysis, the nucleus of our analytical framework for both general mobile ad hoc and wireless sensor networks. We derive expressions of energy consumption for cases with and without perfect wake-up scheme. This comparison is of interest because of the ongoing DoD effort [1] in the development of high precision atomic clock that can be used for synchronized transmission and reception. Two modes of scaling behavior are examined: one increases the node density, the other the network radius, each having its own domain of applications.

The network level analysis for a general mobile ad hoc network which sees substantial link and topological changes is presented in Section IV. The total energy consumption and its scaling behavior are derived for both proactive and reactive networking strategies. The network operation that dominates the energy consumption is identified and the optimal transmission range obtained. Our results also provide the crossover traffic level below which reactive networking is more energy efficient.

We consider next large-scale sensor networks in Section V. Sensor networks are application specific and in many ways different from generic ad hoc networks. Here, we consider two types of applications: the data-centric information retrieval where specific types of data are requested, and the location-centric retrieval in which data from specific locations are needed. We consider two types of sensor network architectures: the multihop ad hoc structure and the sensor network with mobile access (SENMA). Again, we examine scaling behaviors for these two networks using the point-to-point traffic analysis given in Section III. In Section VI, we summarize findings of this paper and comments on some of the limitations of our approach. 


\section{B. Related Work}

The notion of proactive and reactive routing is well known [2]. A new element considered in this paper is the addition of proactive and reactive strategies at the physical layer. For proactive networking, for example, the energy consumed in continuously updating channel states cannot be ignored. Thus, accurate analysis of wireless networks must take into account the energy consumption at the physical layer.

While analytical results on the energy consumption in large-scale ad hoc networks is scarce, many energy aware routing protocols have been proposed, see [3]-[9] and references therein. In [10] and [11], the energy consumption of several well-known routing protocols are studied and compared via simulations. Characterization of the topology of a mobile network is a basic component in network-level analysis on energy consumption. There have been a number of results on the distribution of link distances [12] and the lifetime of a particular route [13], [14] in random multihop networks. In this paper, we obtain an asymptotic characterization of the number of hops in a minimum energy route. This result plays an important role in analyzing the scaling behavior of energy consumption in mobile ad hoc networks.

There is a growing body of literature on wireless sensor networks where the primary concern is energy efficiency. In [15], an analytical approach is taken to characterize energy consumption in sensor networks, and it bears certain similarities to our setup. The analysis in [15], however, differs from ours in both the modeling of energy consumption and the focus of analysis. For example, node listening energy that may dominate the total energy consumption as shown in our study is not included in the analysis of [15]. Furthermore, obtaining scaling behavior is one of the main results of this paper whereas the analysis in [15] does not reveal the scaling law when either the physical size of the network or the density increases. There are also a number of analytical results on sensor network lifetime [16]-[18]. The focus of these results is on the flat ad hoc architecture for sensor networks. In this paper, we compare the performance of the multihop ad hoc architecture with that of SENMA. The performance metric is the scaling behavior of the total energy consumption.

There is an extensive literature discussing proactive, reactive, and hybrid routing protocols [19]-[21]. The energy consumption of the network, however, is usually not the primary metric for comparison. Our results, derived based on generic routing protocols using an idealized network model, focus on the asymptotic behavior of the proactive and reactive strategies and serve as an analytical supplement to the existing practical approaches.

\section{PRoblem Statement}

\section{A. Network Model}

We consider a network with $N$ nodes randomly distributed in a disk of radius $R$. The node distribution is assumed to be uniform with density $\rho=N / 2 \pi R^{2}$. Nodes are half duplex and capable of adjusting the transmission power to cover a neigh- borhood of radius $r .{ }^{1}$ Due to node mobility, the states (whether two nodes are within the transmission range of each other) of communication links vary randomly and asynchronously. We assume homogeneous node mobility and parameterize it by $\lambda_{n}$, the average number of changes in the neighbor set experienced by a node in one unit time. The channel between two mobile nodes is subject to fading with fading rate $\lambda_{c}$, i.e., the channel between two communicating nodes needs to be acquired, on the average, $\lambda_{c}$ times per unit time. A simple example is block fading with coherence time $1 / \lambda_{c}$. By adjusting the relative values of $\lambda_{n}$ and $\lambda_{c}$, we model various fading and mobility conditions.

The message arrival at each node is stationary with mean $\lambda_{m}$ which is referred to as the message duty cycle. Each message contains $L_{m}$ data bits and $L_{c}$ pilot bits for channel estimation and synchronization. If it is necessary to transmit pilot only for the purpose of channel acquisition, we assume that $L_{c}$ pilot bits are transmitted.

\section{B. Radio Model}

When there is no on-going transmission, nodes are in the sleep state by turning off its transceiver. A wake-up scheme is, thus, required to bring nodes to the active communication state when necessary. One approach is to wake up nodes by the RF signals, which can be achieved by equipping each node with an energy detector. In this case, nodes can not be woken up individually; every node within the transmission range $r$ of the transmitting node will be woken up and check whether it is the intended receiver.

A perfect wake-up scheme would be one that brings only the intended receiver back to the active state, thus eliminating unnecessary energy consumption in listening. One possible scheme is to implement a global schedule; nodes are woken up by their internal clock when scheduled for transmission or reception. Another approach is to equip each node with a low-power device such as the remotely activated switch (RAS) [22], [23] enabled by the technology of radio frequency (RF) tags. When the RAS receives a correct paging sequence (for example, a predetermined function of its ID), it turns on the transceiver and brings the node to the active state. In this paper, we study and compare the energy consumption with and without a perfect wake-up scheme.

There are four types of energy consumption at each node: 1 ) when a node is in the sleep state, it consumes $E_{\mathrm{sp}}$ Joules per unit time; 2) when a node is in a "half-awake" state in which the radio of the node is turned on to check if there is an intended message, ${ }^{2}$ it consumes $E_{\text {ha }}$ Joules per bit interval; 3 ) when the node actively decodes information, it consumes receiving energy $E_{\mathrm{rx}}$ Joules/bit; and 4) the transmission of the node that covers a neighborhood of radius $r$ consumes $E_{\mathrm{tx}}(r)$ Joules/bit. From [24]

$$
E_{\mathrm{tx}}(r)=e_{\mathrm{tx}}+\max \left\{e_{\min }, e_{\mathrm{out}} r^{\alpha}\right\}
$$

${ }^{1}$ In our analysis, the transmission range $r$ is optimized for energy efficiency. We do not consider the scenario where the transmission range is selected on a hop-by-hop basis, which requires the knowledge of network topology at each individual node.

${ }^{2}$ This may include searching for a particular transmission signature. 
where $\alpha$ is the path attenuation factor, $e_{\mathrm{tx}}$ the energy consumed by the transmitter circuitry, $e_{\text {out }}$ the antenna output energy to reach, with an acceptable SNR, the destination unit distance away, and $e_{\min }$ the minimum energy radiated regardless of the transmission range. Note that $e_{\min }$ imposes a hard limit on the minimum transmission range

$$
r \geq r_{0} \triangleq\left(\frac{e_{\min }}{e_{\text {out }}}\right)^{\frac{1}{\alpha}}
$$

\section{Performance Measure and Problem Statement}

Our goal is to calculate the overall (average) energy $\mathcal{E}$ consumed in one time unit. This energy is divided into energy $\mathcal{E}_{\mathrm{D}}$ spent in data transmission, energy $\mathcal{E}_{\mathrm{PHY}}$ spent in synchronization and channel acquisition, and energy $\mathcal{E}_{\mathrm{PT}}$ spent in protocol implementation. Energy efficiency $\eta_{\mathrm{D}}$, physical layer overhead $\eta_{\mathrm{PHY}}$, and protocol overhead $\eta_{\mathrm{PT}}$ are defined as, respectively

$$
\eta_{\mathrm{D}}=\frac{\mathcal{E}_{\mathrm{D}}}{\mathcal{E}}, \quad \eta_{\mathrm{PHY}}=\frac{\mathcal{E}_{\mathrm{PHY}}}{\mathcal{E}_{\mathrm{D}}}, \quad \eta_{\mathrm{PT}}=\frac{\mathcal{E}_{\mathrm{PT}}}{\mathcal{E}_{\mathrm{D}}} .
$$

We are interested in evaluating these quantities for proactive and reactive networks as functions of network mobility $\lambda_{n}$, channel fading rate $\lambda_{c}$, message duty cycle $\lambda_{m}$, node density $\rho$, and network geographic size $R$.

\section{POINT-TO-POINT TRAFFIC ANALYSIS WITH OPTIMAL ROUTING}

We now calculate the total energy consumed in moving one bit between a randomly chosen source-destination pair, a basic element in our analysis at the network level. We assume here the minimum energy route from the source to the destination has already been established. The overhead associated with route discovery will be addressed in the next section.

\section{A. Minimum Transmission Range}

In our energy analysis, the transmission range $r$ is optimized for energy efficiency. We now characterize the interval from which $r$ can assume values.

The first constraint on $r$ is the hardware limit given in (2). The second constraint is network connectivity, i.e., $r$ should be large enough so that the network is connected with high probability. Let $r_{c}(N)$ denote the minimum transmission range to ensure connectivity with probability 1 for a network with $N$ uniformly distributed nodes, we have, from [25]

$$
r \geq r_{c}(N) \stackrel{N \rightarrow \infty}{\rightarrow} R \sqrt{\frac{\log N}{N}}=\left\{\begin{array}{ll}
\mathcal{O}\left(\sqrt{\frac{\log N}{N}}\right) & \rho \uparrow \\
\mathcal{O}(\sqrt{\log N}) & R \uparrow
\end{array} .\right.
$$

We see that when the network size $N$ is increased by increasing the node density $\rho$, the minimum transmission range $r_{c}(N)$ eventually goes to 0 . If, however, $N$ is increased by increasing the geographic size $R$, the transmission range has to grow to infinity with rate $\sqrt{\log N}$ to ensure network connectivity. ${ }^{3}$

\footnotetext{
${ }^{3}$ In practice, the transmission range $r$ cannot grow unboundedly. Nevertheless, (3) shows how fast $r$ should increase with $R$ to ensure network connectivity. To study the scaling behavior of the network when $R$ increases, the fact that $r$ should grow with rate $\sqrt{\log N}$ has to be taken into consideration unless the outage of network connectivity is modeled, which is a different framework from what is considered in this paper.
}

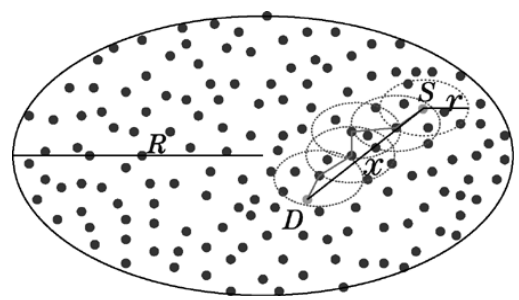

Fig. 1. Point-to-point traffic analysis between source $S$ and destination $D$.

Combining (2) and (3), we obtain the minimum transmission range $r_{\min }$ as

$$
r \geq r_{\min } \triangleq \max \left\{r_{0}, R \sqrt{\frac{\log N}{N}}\right\}
$$

\section{B. Number of Hops}

Let $h(x, r)$ be the number of hops in a minimum energy route for a source-destination pair with distance $x$ and transmission range $r$ (see Fig. 1). Clearly, $h(x, r)$ is a random variable depending on the random node locations. When $N$ is large, however, we can give a probabilistic characterization on $h(x, r)$ as in the following proposition.

Proposition 1: For $r \geq r_{\min }$, the number of hops $h(x, r)$ in a minimum energy route converges to $\lceil x / r\rceil$ in probability as $N \rightarrow \infty$ by increasing either $\rho$ or $R$.

Proof: See Appendix A.

With Proposition 1, we can then study the scaling behavior of energy consumption in large networks using $\lceil x / r\rceil$ as the number of hops.

\section{Energy Consumption With a Perfect Wake-Up Scheme}

With perfect wake-up scheme, only the relaying node listens to the transmission. The energy consumed in one hop comes from one transmission and one reception. Let $X$ denote the distance between the source $S$ and the destination $D$ with probability density function (pdf) $p_{X}(x)$. Let $\mathcal{E}_{\mathrm{S}-\mathrm{D}}$ be the total energy consumed in moving one bit between $S$ and $D$. From Proposition 1 , we have, with probability approaching 1 as $N \rightarrow \infty$

$$
\begin{aligned}
\mathcal{E}_{\mathrm{S}-\mathrm{D}} & =\min _{r \geq r_{\min }} \int_{0}^{2 R}\left(E_{\mathrm{tx}}(r)+E_{\mathrm{rx}}\right) h(x, r) p_{X}(x) \mathrm{d} x \\
& =\min _{r \geq r_{\min }}\left(E_{\mathrm{tx}}(r)+E_{\mathrm{rx}}\right) \frac{\mathbb{E}(X)}{r} \\
& =\min _{r \geq r_{\min }}\left(e_{\mathrm{tx}}+\max \left\{e_{\min }, e_{\mathrm{out}} r^{\alpha}\right\}+E_{\mathrm{rx}}\right) \frac{\mathbb{E}(X)}{r}
\end{aligned}
$$

where for the ease of presentation, we have used $x / r$ instead of $\lceil x / r\rceil$ as the number of hops. Obviously, this approximation does not affect the scaling behavior of the network. It is easy to show that the transmission range $r^{*}$ that minimizes (5) is given by

$$
r^{*}=\max \left\{\left(\frac{E_{\mathrm{rx}}+e_{\mathrm{tx}}}{e_{\mathrm{out}}(\alpha-1)}\right)^{\frac{1}{\alpha}}, r_{\min }\right\} .
$$

We now examine how $\mathcal{E}_{\mathrm{S}-\mathrm{D}}$ scales with the network size $N$. When $N$ is increased by increasing $\rho$, the minimum trans- 


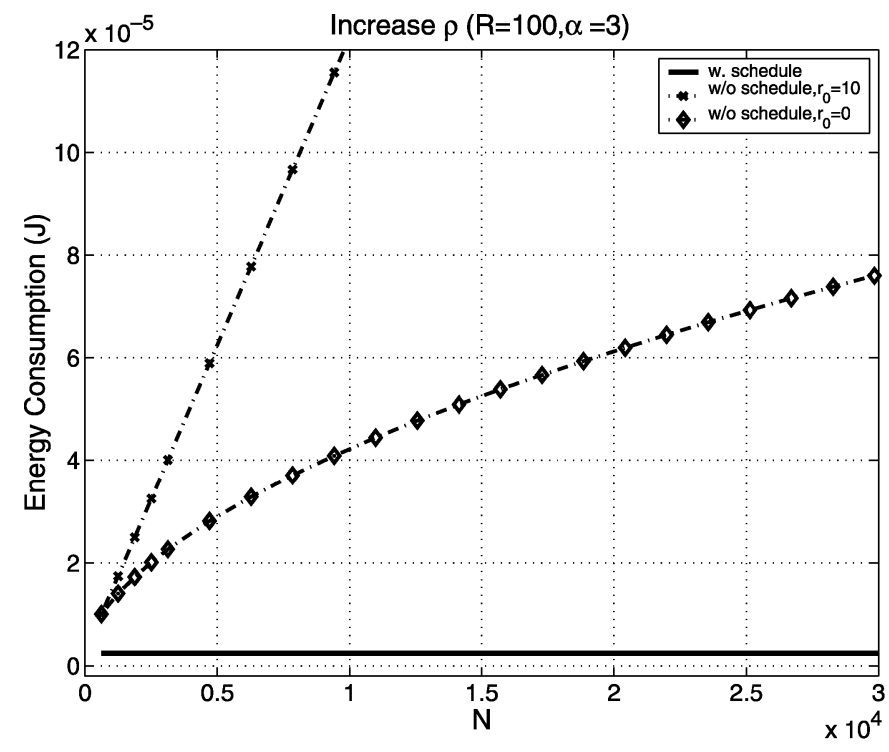

(a)
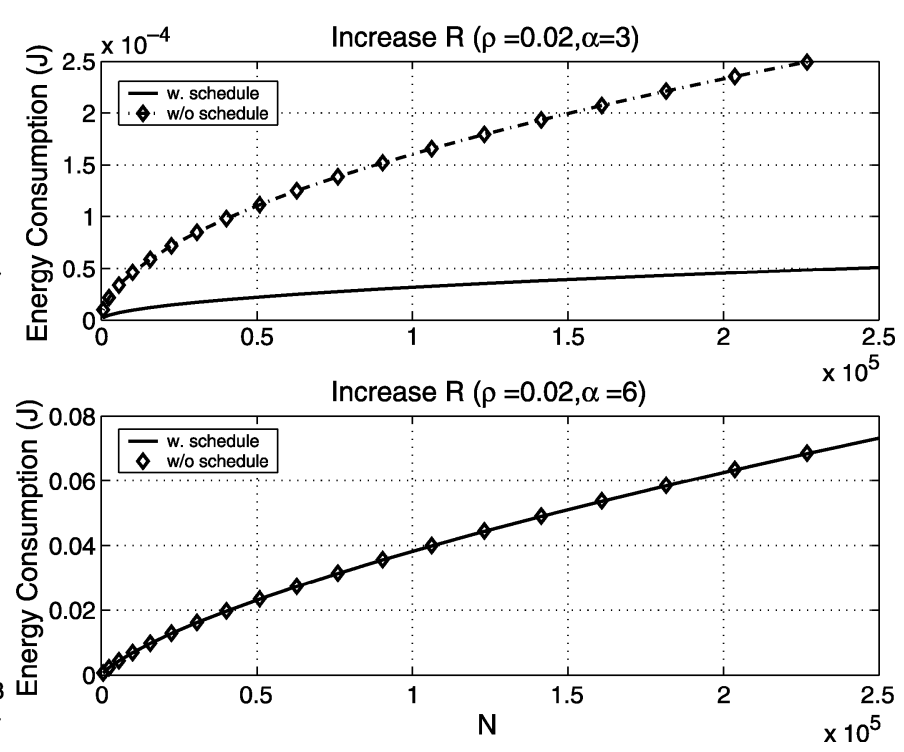

(b)

Fig. 2. Numerical results for point-to-point traffic analysis. (a) Increase $\rho$. (b) Increase $R$. ( $\left.e_{\mathrm{tx}}=81 \mathrm{~nJ}, e_{\mathrm{out}}=0.1 \mathrm{~nJ}, E_{\mathrm{rx}}=180 \mathrm{~nJ}, E_{\mathrm{ha}}=90 \mathrm{~nJ}\right)$.

mission range $R \sqrt{\log N / N}$ to ensure network connectivity approaches to 0 . We thus have, substituting (6) into (5)

$$
\mathcal{E}_{\mathrm{S}-\mathrm{D}} \stackrel{\rho \rightarrow \infty}{\rightarrow}\left\{\begin{array}{cl}
\alpha e_{\text {out }}^{\frac{1}{\alpha}}\left(\frac{e_{\mathrm{tx}}+E_{\mathrm{rx}}}{\alpha-1}\right)^{1-\frac{1}{\alpha}} & \\
\times \mathbb{E}(X)=\mathcal{O}(1), & \text { if }\left(\frac{E_{\mathrm{rx}}+e_{\mathrm{tx}}}{e_{\text {out }}(\alpha-1)}\right)^{\frac{1}{\alpha}}>r_{0} \\
\left(\frac{e_{\text {out }}}{e_{\min }}\right)^{\frac{1}{\alpha}}\left(E_{\mathrm{rx}}+e_{\mathrm{tx}}+e_{\mathrm{min}}\right) & \\
\times \mathbb{E}(X)=\mathcal{O}(1), & \text { otherwise }
\end{array}\right.
$$

where we have used the fact that $\mathbb{E}(X)$ is a constant independent of the network size $N$.

When $N$ is increased by increasing $R$, the optimal transmission range $r^{*}$ is eventually given by $R \sqrt{\log N / N}=$ $\mathcal{O}(\sqrt{\log N})$. We thus have, considering $\mathbb{E}(X)=\mathcal{O}(R)=$ $\mathcal{O}(\sqrt{N})$

$\mathcal{E}_{\mathrm{S}-\mathrm{D}} \stackrel{R \rightarrow \infty}{\rightarrow} e_{\mathrm{out}}\left(R \sqrt{\frac{\log N}{N}}\right)^{\alpha-1} \mathbb{E}(X)=\mathcal{O}\left(\sqrt{N(\log N)^{\alpha-1}}\right)$.

Combining (7) and (8) we obtain the scaling law for the point-topoint transmission with perfect wake-up scheme as

$$
\mathcal{E}_{\mathrm{S}-\mathrm{D}}=\left\{\begin{array}{ll}
\mathcal{O}(1), & \rho \uparrow \\
\mathcal{O}\left(\sqrt{N(\log N)^{\alpha-1}}\right), & R \uparrow
\end{array} .\right.
$$

\section{Energy Consumption Without a Perfect Wake-Up Scheme}

When nodes can not be woken up individually, all neighboring nodes listen to the transmission. The total energy $\overline{\mathcal{E}}_{\mathrm{S}-\mathrm{D}}$ is given by

$$
\begin{aligned}
\overline{\mathcal{E}}_{\mathrm{S}-\mathrm{D}} & =\min _{r \geq r_{\min }}\left(E_{\mathrm{tx}}(r)+E_{\mathrm{rx}}+\frac{r^{2}}{R^{2}}(N-2) E_{\mathrm{ha}}\right) \frac{\mathbb{E}(X)}{r} \\
& = \begin{cases}\mathcal{O}(\sqrt{N \log N}), & \rho \uparrow, r_{0}=0 \\
\mathcal{O}(N), & \rho \uparrow, r_{0}>0 . \\
\mathcal{O}\left(\sqrt{N(\log N)^{\alpha-1}}\right), & R \uparrow\end{cases}
\end{aligned}
$$

In all cases, the optimal transmission range is given by $r_{\min }$ defined in (4). Comparing (9) and (11), we see that when density increases, the energy consumption without a perfect wake-up scheme increases much faster than that with a perfect wake-up scheme. The reason for this is that the listening energy dominates when $\rho$ increases. The use of a prefect wake-up scheme, thus, leads to a different scaling law. On the other hand, when the radius $R$ of the network increases, the energy consumed in transmission dominates, resulting in identical scaling behavior of these two cases.

The different scaling behavior resulted from having a hardware limit on the minimum transmission range provides guidance for transceiver circuit design. Since the energy consumption has a more favorable scaling behavior for $r_{0}=0$ than that for $r_{0}>0$, one should design $r_{0}$ so that it is smaller than the minimum transmission range dictated by the network connectivity condition in order to achieve better energy efficiency.

\section{E. An Example: The Benefit of a Perfect Wake-Up Scheme}

Expressions (5) and (10) allow us to evaluate the benefit of having a perfect wake-up scheme. Using typical values for the radio model given in [24], we calculate the energy consumption as a function of the network size $N$. In Fig. 2(a), the network size $N$ is increased by increasing $\rho$. We can see that a perfect wake-up scheme results in significant improvement in energy efficiency, especially when there is a hardware limit on the transmission range. At $N=1000$, the energy consumption is reduced by about 8 times and at $N=10000,60$ times with a perfect wake-up scheme.

In Fig. 2(b), we show the energy consumption when $N$ is increased by increasing $R$. In the upper plot, we set $\alpha=3$; in the lower one, $\alpha=6$. We see that although the use of a perfect wake-up scheme does not change the scaling behavior of the energy consumption, when $\alpha$ is small, it does make a difference 
for finite $N$; at $N=1000$, the energy consumption is reduced by five times. When $\alpha=6$, having a perfect wake-up scheme has little effect on the total energy consumption. This result can be explained by examining (10). Without a perfect wake-up scheme, the listening energy scales with $\sqrt{N \log N}$ while the transmitting energy scales with $\sqrt{N(\log N)^{\alpha-1}}$. When $\alpha$ is small, the difference between the listening energy and the transmitting energy is small for relatively small $N$. Thus, the listening energy can significantly increase the total energy, resulting in higher energy expenditure as compared with that of using a perfect wake-up scheme.

\section{NETWORK TRAFFIC ANALYSIS}

In this section, we analyze the energy efficiency of the proactive and reactive networking by taking into account both the protocol overhead and the physical layer overhead.

\section{A. Energy Consumption for Reactive Networking}

The total energy consumption is divided into four parts: route discovery, channel acquisition and synchronization, message transmission, and sleeping. We calculate them separately as follows.

1) Route Discovery: For reactive networking, route needs to be established before message transmission. We consider a hop-by-hop routing protocol similar to ad hoc on-demand distance vector (AODV) [26].

As illustrated in Fig. 3, when source $S$ has a message for $D$, it initiates a route discovery process by broadcasting a request to its neighbors. The request packet contains a hop count and the addresses of the source and the destination. A neighboring node decodes the request packet, replaces the source address with its own, sets a reverse pointer to the transmitting node, increases the hop count by one, and broadcasts the new request packet. In Fig. 3, two different routes from $S$ to $D$ are illustrated.

When the request packets reach the destination, $D$ chooses the node, say $A$, whose request packet has the smallest hop count and transmits a reply. Upon receiving the reply, $A$ sets a pointer to $D$ and transmits a reply packet to $B$ - the node from which $A$ receives the request with the smallest hop count. This process continues until the reply reaches the source $S$. Now, a route from $S$ to $D$ has been established.

We now compute the total energy consumed in route discovery in one unit time within which total $\lambda_{m} N$ (on the average) messages are generated. For large network $(N \rightarrow \infty)$ with uniform traffic and fixed message arrival rate $\lambda_{m}$, the probability that a route established for one message can be used for another message approaches 0 as $N$ goes to infinity. ${ }^{4}$ Thus, on the average, $\lambda_{m} N$ route discovery processes are initiated within one

\footnotetext{
${ }^{4} \mathrm{We}$ do not consider cache and local route repair. The probability that a route established for one message can be used for another is, thus, the probability that two messages are generated for the same source-destination pair before the route becomes invalid due to topology change. It is easy to show that the probability that two messages are generated for the same source-destination pair within a time period of $T$ is $N\left(_{m_{m} T}\right)((N-1) / N)^{\lambda_{m} T-2}(1 / N)^{2}$ which approaches 0 as $N \rightarrow \infty$ for any $T>^{2} 0$. Thus, for a mobile network with zero probability of being stationary, the probability that a route established for one message can be used for another approaches 0 as $N \rightarrow \infty$.
}

unit of time. The total energy consumed in route discovery in the presence of a perfect wake-up scheme is given by

$$
\begin{aligned}
\mathcal{E}_{\mathrm{RN}, \mathrm{r}}(r)=\lambda_{m} N\{ & (N-1)\left(2\lceil\log N\rceil+\left\lceil\log \left(\frac{2 R}{r}\right)\right\rceil\right) \\
& \times\left(E_{\mathrm{tx}}(r)+\frac{r^{2}}{R^{2}}(N-1) E_{\mathrm{rx}}\right) \\
& \left.+2\lceil\log N\rceil \frac{\mathbb{E}(X)}{r}\left(E_{\mathrm{tx}}(r)+E_{\mathrm{rx}}\right)\right\}
\end{aligned}
$$

where $\mathbb{E}(X)$ denotes the average distance between a randomly chosen source-destination pair. The first term is the energy consumed in the transmission of requests: $2\lceil\log N\rceil$ is the number of bits for addresses and $\lceil\log (2 R / r)\rceil$ for hop counts. Every node transmits once with energy $E_{\mathrm{tx}}(r)$ in the flood of the request. For each transmission, all neighboring nodes decode, consuming energy $\left(r^{2} / R^{2}\right)(N-1) E_{\mathrm{rx}}$. The second term is the energy consumed in the transmission of replies: $2\lceil\log N\rceil$ is the number of bits for the addresses of the destination and the transmitting node, ${ }^{5} E_{\mathrm{tx}}(r)+E_{\mathrm{rx}}$ the transmitting and listening energy in one hop, and $\mathbb{E}(X) / r$ the asymptotic average number of hops to reach the source $S$.

Without a perfect wake-up scheme, all neighbors of the chosen relaying nodes listen to the transmission of replies. The energy consumed in route discovery is given by

$$
\begin{aligned}
\overline{\mathcal{E}}_{\mathrm{RN}, \mathrm{r}}(r) & =\lambda_{m} N\{ \\
= & (N-1)\left(2\lceil\log N\rceil+\left\lceil\log \left(\frac{2 R}{r}\right)\right\rceil\right) \\
& \times\left(E_{\mathrm{tx}}(r)+\frac{r^{2}}{R^{2}}(N-1) E_{\mathrm{rx}}\right)+3\lceil\log N\rceil \frac{\mathbb{E}(X)}{r} \\
& \left.\times\left(E_{\mathrm{tx}}(r)+E_{\mathrm{rx}}+\frac{r^{2}}{R^{2}}(N-2) E_{\mathrm{ha}}\right)\right\}
\end{aligned}
$$

2) Physical Layer Overhead in Channel Acquisition: With a perfect wake-up scheme, we have

$$
\begin{gathered}
\mathcal{E}_{\mathrm{RN}, \mathrm{c}}(r)=\lambda_{m} N\left\{(N-1) L_{c}\left(E_{\mathrm{tx}}(r)+\frac{r^{2}}{R^{2}}(N-1) E_{\mathrm{rx}}\right)\right. \\
\left.+L_{c} \frac{\mathbb{E}(X)}{r}\left(E_{\mathrm{tx}}(r)+E_{\mathrm{rx}}\right)\right\}
\end{gathered}
$$

where the first term is the energy for training bits embedded in the flooding of request packets during route discovery where all neighbors decode, the second is the energy for channel estimation during message transmission where only the intended receiver listens with the use of a perfect wake-up scheme. ${ }^{6}$ Similarly, the energy consumed in channel acquisition without a perfect wake-up scheme is

$$
\begin{aligned}
\overline{\mathcal{E}}_{\mathrm{RN}, \mathrm{c}}(r)= & \lambda_{m} N\left\{(N-1) L_{c}\left(E_{\mathrm{tx}}(r)+\frac{r^{2}}{R^{2}}(N-1) E_{\mathrm{rx}}\right)\right. \\
& \left.+L_{c} \frac{\mathbb{E}(X)}{r}\left(E_{\mathrm{tx}}(r)+E_{\mathrm{rx}}+\frac{r^{2}}{R^{2}}(N-2) E_{\mathrm{ha}}\right)\right\} .
\end{aligned}
$$

${ }^{5}$ With a perfect wake-up scheme, the address of the receiving node does not need to be specified; the node that is woken up is the intended receiver.

${ }^{6}$ For simplicity, we have assumed that the bandwidth is large relatively to the packet length so that the packet transmission time is no larger than the channel coherence time $1 / \lambda_{c}$. It is straightforward to extend the analysis to scenarios where channel acquisition has to be carried out more than once during message transmission. 


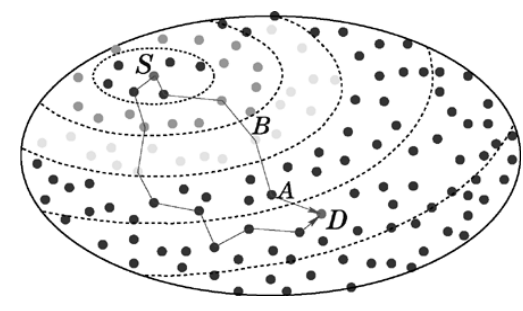

Fig. 3. Hop-by-hop route discovery in reactive networking.

3) Message Transmission: Energy consumption in message transmission with a perfect wake-up scheme has the same expression as in the point-to-point traffic analysis given in (5). The only difference is that the optimization over the transmission range $r$ should be carried out on the total energy consumption including the overhead associated with route discovery and channel acquisition. Since the information on the relaying node is provided by the perfect wake-up scheme (the node that is woken up is the relaying node), only the addresses of the source and the destination need to be embedded in the message

$$
\begin{aligned}
\mathcal{E}_{\mathrm{RN}, \mathrm{m}}(r)= & \lambda_{m} N\left(L_{m}+2\lceil\log N\rceil\right)\left(E_{\mathrm{tx}}(r)+E_{\mathrm{rx}}\right) \frac{\mathbb{E}(X)}{r} \\
= & \underbrace{\lambda_{m} N\left(2\lceil\log N\rceil\left(E_{\mathrm{tx}}(r)+E_{\mathrm{rx}}\right)\right) \frac{\mathbb{E}(X)}{r}}_{\mathcal{E}_{\mathrm{RN}, \mathrm{PT}}(r)} \\
& +\underbrace{\lambda_{m} N L_{m}\left(E_{\mathrm{tx}}(r)+E_{\mathrm{rx}}\right) \frac{\mathbb{E}(X)}{r}}_{\mathcal{E}_{\mathrm{RN}, \mathrm{D}}(r)}
\end{aligned}
$$

where $\mathcal{E}_{\mathrm{RN}, \mathrm{D}}(r)$ denotes the net energy consumed in message transmission, and $\mathcal{E}_{\mathrm{RN}, \mathrm{PT}}(r)$ the protocol overhead in message transmission. Similarly, without a perfect wake-up scheme, we have (16) shown at the bottom of the page.

4) Total Energy Consumption, Overhead, and Efficiency: The total energy consumption $\mathcal{E}_{\mathrm{RN}}$ for reactive networking with a perfect wake-up scheme, optimized over $r$, is given by

$$
\mathcal{E}_{\mathrm{RN}}=\min _{r \geq r_{\text {min }}}\left(\mathcal{E}_{\mathrm{RN}, \mathrm{r}}(r)+\mathcal{E}_{\mathrm{RN}, \mathrm{c}}(r)+\mathcal{E}_{\mathrm{RN}, \mathrm{m}}(r)\right)+N E_{\mathrm{sp}}
$$

where $\mathcal{E}_{\mathrm{RN}, \mathrm{r}}(r), \mathcal{E}_{\mathrm{RN}, \mathrm{c}}(r)$, and $\mathcal{E}_{\mathrm{RN}, \mathrm{m}}(r)$ are given by (12), (14), and (15), respectively. Comparing (12), (14), and (15), we see that the energy consumed in route discovery, specifically, the flooding of the request packets, dominates when the network size $N$ becomes large by increasing either $\rho$ or $R$. We then obtain the following scaling law of the total energy consumption in reactive networking with a perfect wake-up scheme

$$
\mathcal{E}_{\mathrm{RN}}= \begin{cases}\mathcal{O}\left(N^{2}(\log N)^{2}\right), & \rho \uparrow, r_{0}=0 \\ \mathcal{O}\left(N^{3} \log N\right), & \rho \uparrow, r_{0}>0 . \\ \mathcal{O}\left(N^{2} \sqrt{(\log N)^{\alpha+2}}\right), & R \uparrow\end{cases}
$$

In all cases, the optimal transmission range $r^{*}$ is given by $r_{\text {min }}$ when the network size is large. The energy efficiency, the protocol overhead, and the physical layer overhead are, respectively

$$
\begin{aligned}
\eta_{\mathrm{RN}, \mathrm{D}} & =\frac{\mathcal{E}_{\mathrm{RN}, \mathrm{D}}\left(r^{*}\right)}{\mathcal{E}_{\mathrm{RN}}} \\
\eta_{\mathrm{RN}, \mathrm{PHY}} & =\frac{\mathcal{E}_{\mathrm{RN}, \mathrm{c}}\left(r^{*}\right)}{\mathcal{E}_{\mathrm{RN}, \mathrm{D}}\left(r^{*}\right)} \\
\eta_{\mathrm{RN}, \mathrm{PT}} & =\frac{\mathcal{E}_{\mathrm{RN}, \mathrm{r}}\left(r^{*}\right)+\mathcal{E}_{\mathrm{RN}, \mathrm{PT}}\left(r^{*}\right)}{\mathcal{E}_{\mathrm{RN}, \mathrm{D}}\left(r^{*}\right)}
\end{aligned}
$$

where $\mathcal{E}_{\mathrm{RN}, \mathrm{D}}$ and $\mathcal{E}_{\mathrm{RN}, \mathrm{PT}}$ are defined in (15).

Similarly, without a perfect wake-up scheme, we have

$$
\begin{aligned}
\overline{\mathcal{E}}_{\mathrm{RN}} & =\min _{r \geq r_{\min }\left(\overline{\mathcal{E}}_{\mathrm{RN}, \mathrm{r}}(r)+\overline{\mathcal{E}}_{\mathrm{RN}, \mathrm{c}}(r)+\overline{\mathcal{E}}_{\mathrm{RN}, \mathrm{m}}(r)\right)+N E_{\mathrm{sp}}} \\
& = \begin{cases}\mathcal{O}\left(N^{2}(\log N)^{2}\right), & \rho \uparrow, r_{0}=0 \\
\mathcal{O}\left(N^{3} \log N\right), & \rho \uparrow, r_{0}>0 . \\
\mathcal{O}\left(N^{2} \sqrt{(\log N)^{\alpha+2}}\right), & R \uparrow\end{cases}
\end{aligned}
$$

In all cases, the optimal transmission range $r^{*}$ is given by $r_{\min }$ when the network size is large. The energy efficiency, protocol overhead, and physical layer overhead can be similarly obtained.

Comparing (18) and (21), we see that the use of a perfect wake-up scheme does not affect the scaling behavior of reactive networking. The reason is that the dominating factor in energy consumption is the flooding of the request packets in route discovery which requires all neighbors to listen. With and without a perfect wake-up scheme lead to the same scaling law.

\section{B. Energy Consumption for Proactive Networking}

Energy consumption in proactive networking comes from the same four categories as in the reactive networking.

1) Route Maintenance: With proactive networking, network topology and route information are maintained regardless of the message arrivals. We consider here the standard link state routing strategy. Specifically, each node periodically broadcasts a "hello" message (for example, its own ID) to its neighbors.

$$
\begin{aligned}
\overline{\mathcal{E}}_{\mathrm{RN}, \mathrm{m}}(r) & =\lambda_{m} N\left(L_{m}+3\lceil\log N\rceil\right)\left(E_{\mathrm{tx}}(r)+E_{\mathrm{rx}}+\frac{r^{2}}{R^{2}}(N-2) E_{\mathrm{ha}}\right) \frac{\mathbb{E}(X)}{r} \\
& =\underbrace{\lambda_{m} N\left(3\lceil\log N\rceil\left(E_{\mathrm{tx}}(r)+E_{\mathrm{rx}}\right)+\left(L_{m}+3\lceil\log N\rceil\right) \frac{r^{2}}{R^{2}}(N-2) E_{\mathrm{ha}}\right) \frac{\mathbb{E}(X)}{r}}_{\mathcal{E}_{\mathrm{RN}, \mathrm{PT}}(r)}+\underbrace{\lambda_{m} N L_{m}\left(E_{\mathrm{tx}}(r)+E_{\mathrm{rx}}\right) \frac{\mathbb{E}(X)}{r}}_{\mathcal{E}_{\mathrm{RN}, \mathrm{D}}(r)}
\end{aligned}
$$


Every node uses this "hello" message to maintain its neighbor set. Once a node detects a change in its neighbor set, it floods the new neighbor set over the network. Assume that the "hello" message is transmitted at the rate $\left(\lambda_{n}\right)$ of the topology change, we have

$$
\begin{gathered}
\mathcal{E}_{\mathrm{PN}, \mathrm{r}}(r)=N \lambda_{n}\lceil\log N\rceil\left(E_{\mathrm{tx}}(r)+\frac{r^{2}}{R^{2}}(N-1) E_{\mathrm{rx}}\right)+N^{2} \lambda_{n} \\
\times\lceil\log N\rceil \frac{r^{2}}{R^{2}}(N-1)\left(E_{\mathrm{tx}}(r)+\frac{r^{2}}{R^{2}}(N-1) E_{\mathrm{rx}}\right)
\end{gathered}
$$

where the first term is the energy consumed in the transmission of "hello" messages: $\lceil\log N\rceil$ is the length of the message, and every "hello" message is decoded by all the neighboring nodes. The second term is the energy consumed in the flooding of neighbor sets: $\lceil\log N\rceil\left(r^{2} / R^{2}\right)(N-1)$ is the average number of bits to represent the neighbor set; each node floods, on the average, $\lambda_{n}$ times per unit time where each flood consists of $N$ transmissions and every transmission is decoded by all the neighbors. Note that the energy consumed in this proactive route discovery is independent of the wake-up scheme.

2) Physical Layer Overhead in Channel Acquisition: The cost of channel estimation consists of two parts. The first part occurs during the flooding of neighbor sets and the second part during the message transmission. With a perfect wake-up scheme, the total energy consumed in channel acquisition is given by

$$
\begin{aligned}
\mathcal{E}_{\mathrm{PN}, c}(r)=N^{2} \lambda_{n} L_{c} & \left(E_{\mathrm{tx}}(r)+\frac{r^{2}}{R^{2}}(N-1) E_{\mathrm{rx}}\right) \\
& +\lambda_{c} L_{c}\left(E_{\mathrm{tx}}(r)+E_{\mathrm{rx}}\right) \frac{\mathbb{E}(X)}{r} \lambda_{m} N
\end{aligned}
$$

where $\lambda_{m} N$ is the average number of messages generated per unit time, and $(\mathbb{E}(X) / r) \lambda_{m} N$ is the average number of communication links that need to be updated. Similarly, without a perfect wake-up scheme, we have

$$
\begin{aligned}
& \overline{\mathcal{E}}_{\mathrm{PN}, \mathrm{c}}(r)=N^{2} \lambda_{n} L_{c}\left(E_{\mathrm{tx}}(r)+\frac{r^{2}}{R^{2}}(N-1) E_{\mathrm{rx}}\right) \\
& +\lambda_{c} L_{c}\left(E_{\mathrm{tx}}(r)+E_{\mathrm{rx}}+\frac{r^{2}}{R^{2}}(N-2) E_{\mathrm{ha}}\right) \frac{\mathbb{E}(X)}{r} \lambda_{m} N .
\end{aligned}
$$

3) Message Transmission: The analysis is the same as that for reactive networking, as shown in (24) and (25) at the bottom of the page.

4) Total Energy Consumption, Overhead, and Efficiency: We now compute the total energy $\mathcal{E}_{\mathrm{PN}}$ consumed in one unit time. With a perfect wake-up scheme, the total energy is given by

$$
\mathcal{E}_{\mathrm{PN}}=\min _{r \geq r_{\mathrm{min}}}\left(\mathcal{E}_{\mathrm{PN}, \mathrm{r}}(r)+\mathcal{E}_{\mathrm{PN}, \mathrm{c}}(r)+\mathcal{E}_{\mathrm{PN}, \mathrm{m}}(r)\right)+N E_{\mathrm{sp}}
$$

We now examine the scaling behavior of $\mathcal{E}_{\mathrm{PN}}$. Comparing (22)-(24), we see that the energy consumed in route maintenance, specifically, the flooding of the updated neighbor set dominates when $N \rightarrow \infty$ by increasing either $\rho$ or $R$. We then obtain the scaling law as

$$
\mathcal{E}_{\mathrm{PN}}=\left\{\begin{array}{ll}
\mathcal{O}\left(N^{2}(\log N)^{3}\right), & \rho \uparrow, r_{0}=0 \\
\mathcal{O}\left(N^{4} \log N\right), & \rho \uparrow, r_{0}>0 \\
\mathcal{O}\left(N^{2} \sqrt{(\log N)^{\alpha+4}}\right), & R \uparrow
\end{array} .\right.
$$

In all cases, the optimal transmission range for large $N$ is given by $r_{\min }$. Similarly, the total energy $\overline{\mathcal{E}}_{\mathrm{PN}}$ consumed in one unit time without a perfect wake-up scheme is given by

$$
\begin{aligned}
\overline{\mathcal{E}}_{\mathrm{PN}} & =\min _{r \geq r_{\min }}\left(\mathcal{E}_{\mathrm{PN}, \mathrm{r}}(r)+\overline{\mathcal{E}}_{\mathrm{PN}, \mathrm{c}}(r)+\overline{\mathcal{E}}_{\mathrm{PN}, \mathrm{m}}(r)\right)+N E_{\mathrm{sp}} \\
& = \begin{cases}\mathcal{O}\left(N^{2}(\log N)^{3}\right), & \rho \uparrow, r_{0}=0 \\
\mathcal{O}\left(N^{4} \log N\right), & \rho \uparrow, r_{0}>0 \\
\mathcal{O}\left(N^{2} \sqrt{(\log N)^{\alpha+4}}\right), & R \uparrow\end{cases}
\end{aligned}
$$

The optimal transmission range for large $N$ is again given by $r_{\min }$. Since the dominating factor is the flooding of the updated neighbor set which requires the listening of all neighbors, we see the same scaling behavior for cases with and without a perfect wake-up scheme. The energy efficiency, protocol overhead, and physical layer overhead can be similarly obtained.

\section{Proactive Versus Reactive Networking}

We now compare proactive networking and reactive networking in terms of energy efficiency. The values for the radio model are the same as used to obtain Fig. 2. Shown in Fig. 4(a) is the total energy consumption and Fig. 4(b) the energy efficiency. We point out that due to the sleep energy, the total energy consumption of the reactive networking levels off at low message duty cycle.

Orders of magnitude of improvement can be achieved by reactive networking when the message duty cycle is low. When the message duty cycle is high, however, proactive networking is more efficient. From (17) and (26), we can solve for the crossover point $\lambda_{0}$ below which the reactive approach is desired over the proactive approach in the presence of a perfect wake-up scheme. Similarly, the crossover point $\bar{\lambda}_{0}$ without a perfect wake-up scheme can be obtained from (20) and (28).

Shown in Fig. 5 is the crossover point as a function of the network mobility. As expected, the smaller the topology change

$$
\begin{aligned}
& \mathcal{E}_{\mathrm{PN}, \mathrm{m}}(r)=\underbrace{L_{m}\left(E_{\mathrm{tx}}(r)+E_{\mathrm{rx}}\right) \frac{\mathbb{E}(X)}{r}}_{\mathcal{E}_{\mathrm{PN}, \mathrm{D}}(r)}+\underbrace{2\lceil\log N\rceil\left(E_{\mathrm{tx}}(r)+E_{\mathrm{rx}}\right) \frac{\mathbb{E}(X)}{r}}_{\mathcal{E}_{\mathrm{PN}, \mathrm{PT}}(r)} \\
& \overline{\mathcal{E}}_{\mathrm{PN}, \mathrm{m}}(r)=\underbrace{\left(3\lceil\log N\rceil\left(E_{\mathrm{tx}}(r)+E_{\mathrm{rx}}\right)+\frac{r^{2}}{R^{2}}\left(L_{m}+3\lceil\log N\rceil\right)(N-2) E_{\mathrm{ha}}\right) \frac{\mathbb{E}(X)}{r}}_{\mathcal{E}_{\mathrm{PN}, \mathrm{PT}}(r)}+\underbrace{L_{m}\left(E_{\mathrm{tx}}(r)+E_{\mathrm{rx}}\right) \frac{\mathbb{E}(X)}{r}}_{\mathcal{E}_{\mathrm{PN}, \mathrm{D}}(r)}
\end{aligned}
$$




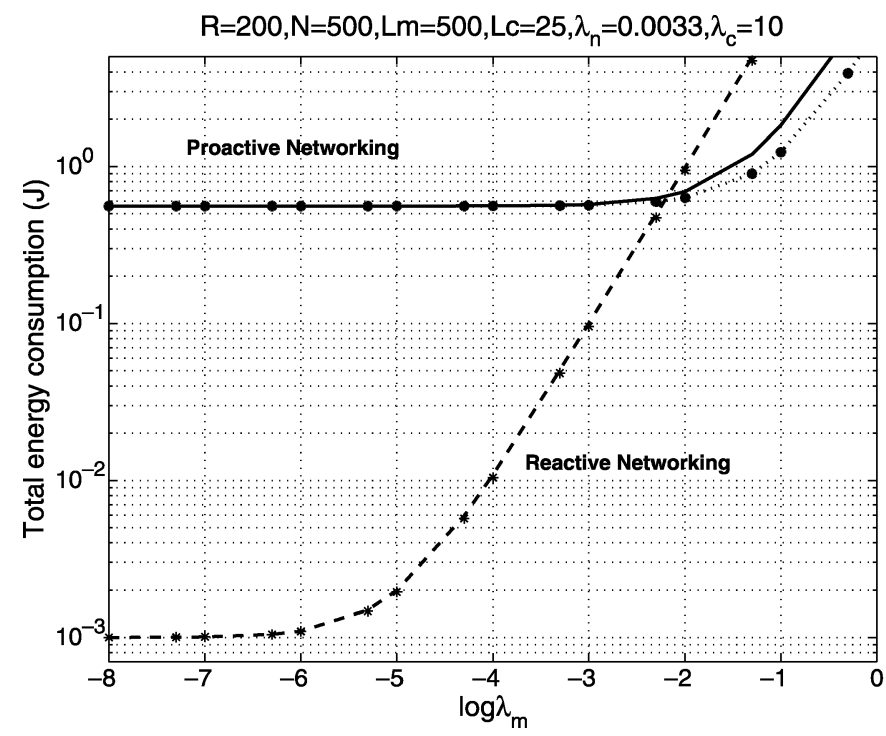

(a)

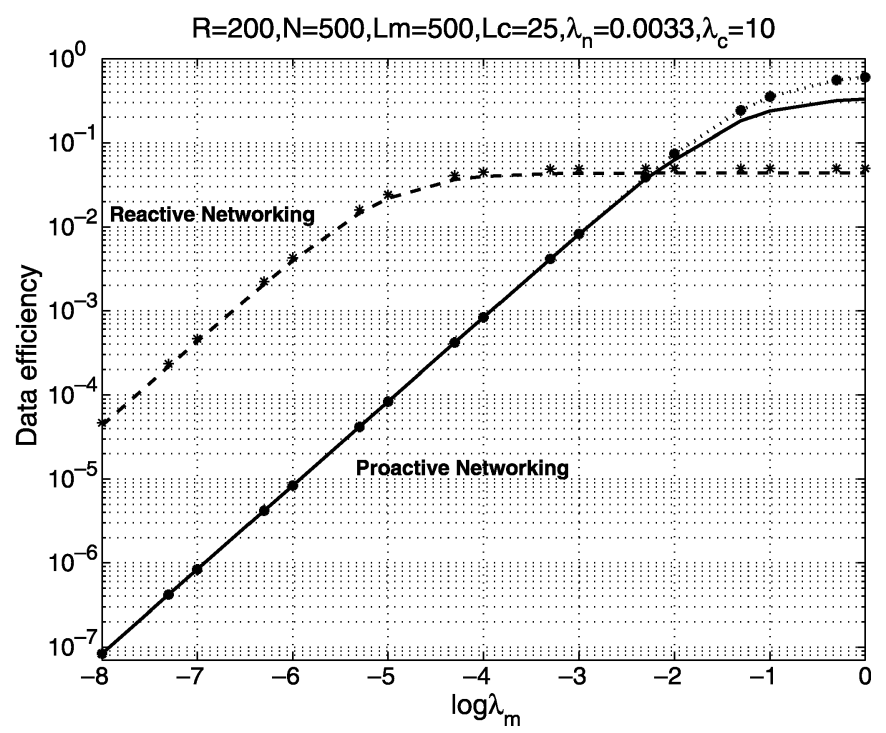

(b)

Fig. 4. Total energy consumption and energy efficiency versus message duty cycle $\lambda_{m}$ (“* $*$ :"with perfect wake-up scheme, “-:” without perfect wake-up scheme).

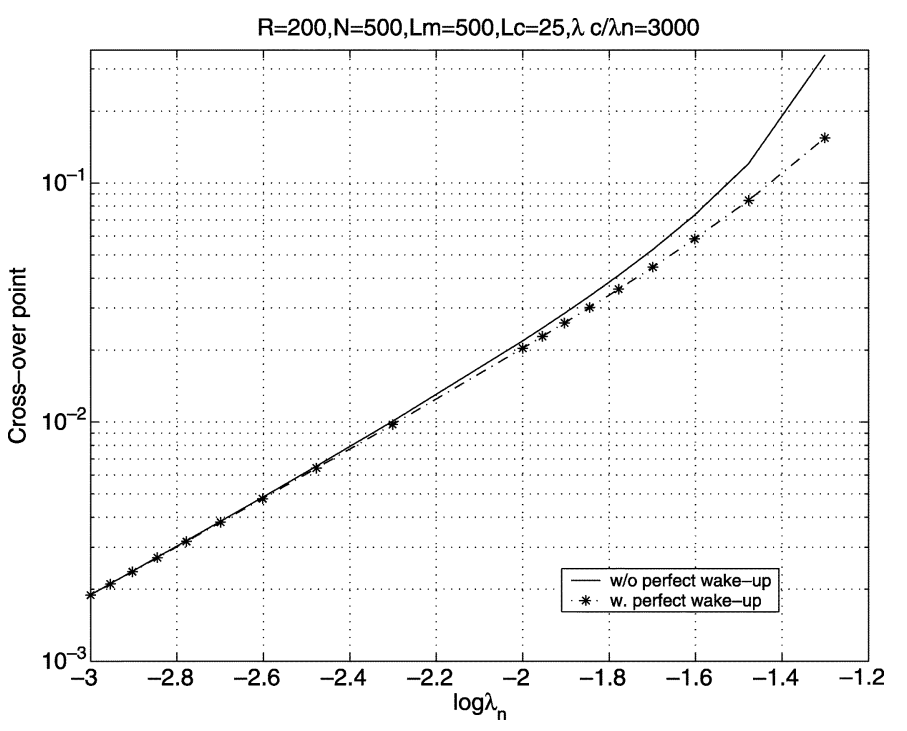

Fig. 5. Crossover point as a function of network mobility.

rate $\lambda_{n}$, the smaller the crossover point $\lambda_{0}$. In other words, the region where the reactive approach is desired becomes smaller when the network mobility is low.

Shown in Fig. 6(a) is the protocol overhead and in Fig. 6(b) the physical layer overhead. We can see that the use of a perfect wake-up scheme results in a substantial reduction in protocol overhead, especially at high message arrival rate. For the physical layer overhead, perfect scheduling provides minimal improvement. Reactive networking, however, provides significant reduction in the physical layer overhead at low message duty cycle.

\section{ENERGY EFFICIENCY OF LARGE-SCALE SENSOR NETWORKS}

We consider in this section an analysis of energy consumption in large-scale sensor networks. We focus on query-reply operations where specific types of data are requested and gathered. In particular, we consider two types of queries: data-centric and location-centric queries. Data-centric query means that a specific type of data (not necessarily the origin of the data) are requested. For example, it may be desirable to collect from those nodes who have measurements with certain characteristics (e.g., temperature measurements higher than $100^{\circ} \mathrm{F}$ ). In such cases, the access point (gateway node or mobile access point) does not know the locations where the requested data reside, thus, must flood the request throughout the network. For location-centric query, in contrast, the access point collects data from specific locations therefore knows the general location where data reside.

\section{A. Sensor Network Architecture: Ad Hoc Sensor Networks}

The prevailing architecture for sensor networks is the multihop ad hoc structure as shown in Fig. 7(a), in which sensors propagate data in multiple hops toward gateway nodes. We assume that sensors are uniformly distributed, and there is one gateway node located at the center of a disk of radius $R$.

While the ad hoc sensor network appears to have almost the identical architecture as that of the ad hoc network considered in previous sections, there are several important differences that will affect the energy analysis. First, for query-reply operations, traffic in sensor networks flows asymmetrically, toward or away from gateway nodes. Second, sensors and gateway nodes are stationary, and topological changes are minimal, i.e., the topology change rate $\lambda_{n}$ is much smaller than the message duty cycle $\lambda_{m}$. The implication is that route discovery needs to be performed only once, after which all sensors know the route to the gateway node. ${ }^{7}$ Finally, it is perhaps unrealistic to assume that, in a large-scale network of low cost sensors, nodes have a perfect wake-up scheme.

\section{B. Sensor Network Architecture: SENMA}

An alternative architecture is SENMA [27], [28] illustrated in Fig. 7(b). SENMA has two types of nodes: a large number of

${ }^{7}$ This is a favorable assumption for ad hoc architecture. In practice, sensor network topology may change since sensor nodes may not function due to a variety of reasons. 


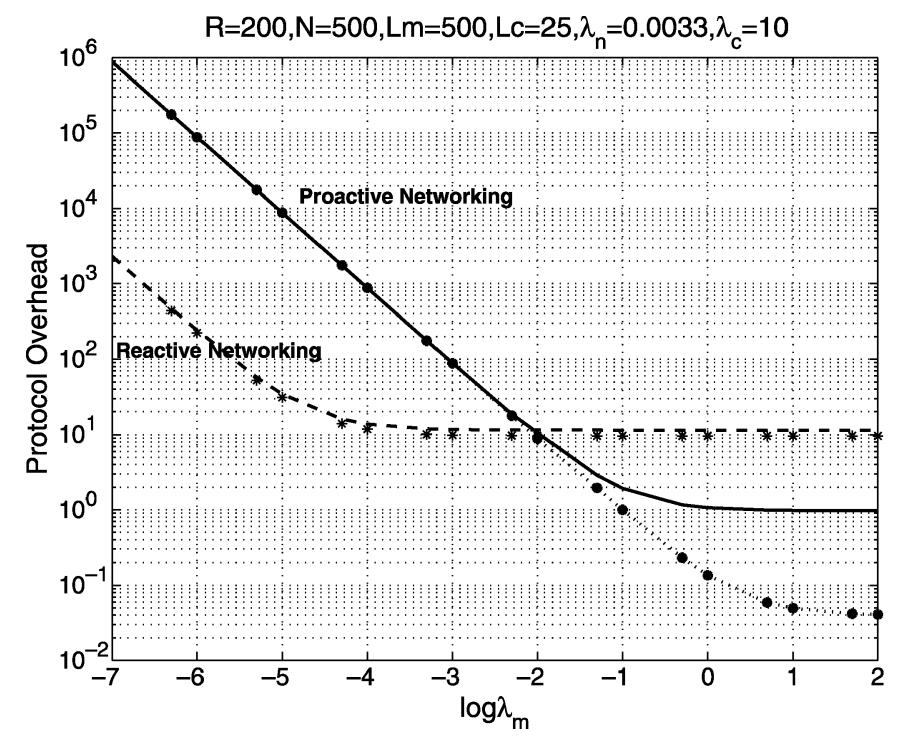

(a)

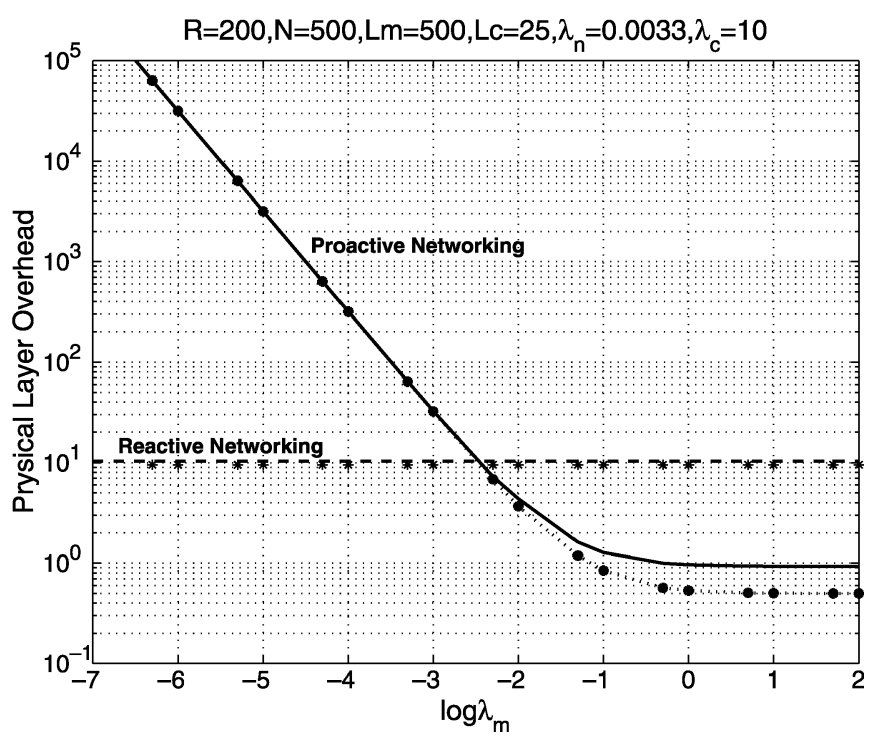

(b)

Fig. 6. Protocol overhead and physical layer overhead versus message duty cycle $\lambda_{m}$ (“*:” with perfect wake-up scheme, “-:" without perfect wake-up scheme).

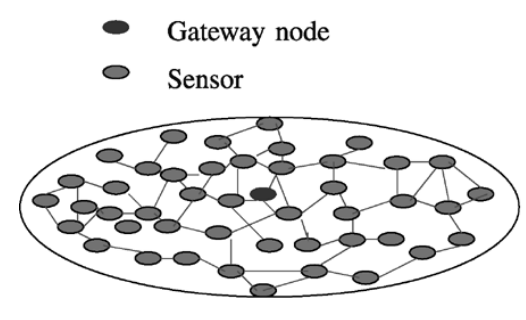

(a)

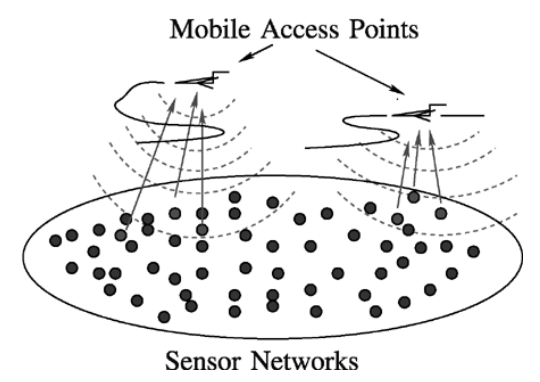

(b)

Fig. 7. Sensor network architectures. (a) Ad hoc sensor networks with gateway nodes. (b) Sensor networks with mobile access (SENMA).

stationary low-power sensor nodes and a small number of powerful mobile access points (APs). Mobile access points may be as sophisticated as UAVs and UGVs or as simple as balloons or even trained dogs or doves carrying transceivers. The premise of SENMA is to move energy consuming tasks away from sensors to mobile APs which, presumably, are not energy constrained. Under SENMA, the sensors communicate directly with the mobile AP in the query-reply operations.

A few caveats are necessary to put the comparison between these two architectures in perspectives. Since our focus is on the energy consumption of the sensors, the energy consumed by the mobile AP and the gateway node will not be included. Unlike in ad hoc networks where we allow each node to adjust its transmission power to minimize energy consumption, which may lead to an arbitrarily small transmission range $r$, we will exclude the possibility that the mobile AP can be arbitrarily close to a sensor. The mobility of the AP is another factor needs to be considered. We assume that the mobile AP can position itself at specific locations and activate sensors in its neighborhood of radius $d$.

\section{Energy Efficiency for Data-Centric Applications}

We first consider data-centric applications. Under the flat ad hoc architecture, the gateway node first broadcasts (in one transmission) the request over the network. Sensors with the requested data then start to transmit to the gateway node using predetermined routes. Assume that a fraction $\beta$ of sensors have the requested data, and they are uniformly distributed in the network. We obtain the total energy consumed in completing this request as

$$
\begin{aligned}
\mathcal{E}_{\text {AdHoc }}^{(d)}= & N E_{\mathrm{rx}}+\beta N \\
& \times \min _{r \geq r_{\text {min }}}\left(E_{\mathrm{rx}}+E_{\mathrm{tx}}(r)+\frac{r^{2}}{R^{2}}(N-2) E_{\mathrm{ha}}\right) \frac{\mathbb{E}(X)}{r} \\
= & \begin{cases}\mathcal{O}\left(\sqrt{N^{3} \log N}\right), & \rho \uparrow, r_{0}=0 \\
\mathcal{O}\left(N^{2}\right), & \rho \uparrow, r_{0}>0 \\
\mathcal{O}\left(\sqrt{N^{3}(\log N)^{\alpha-1}}\right), & R \uparrow\end{cases}
\end{aligned}
$$

where for simplicity, we have omitted the energy consumption for channel acquisition and assumed that both the request and the data from sensors contain only $1 \mathrm{bit}$.

In SENMA, the mobile AP scans the sensor field and broadcasts the request. Activated sensors with the requested data then transmit directly to the mobile AP. Assume that the mobile AP is $H$ away from the activated sensors, we have

$$
\mathcal{E}_{\text {SENMA }}^{(d)}=N E_{\mathrm{rx}}+\beta N E_{\mathrm{tx}}(H)=\mathcal{O}(N) .
$$




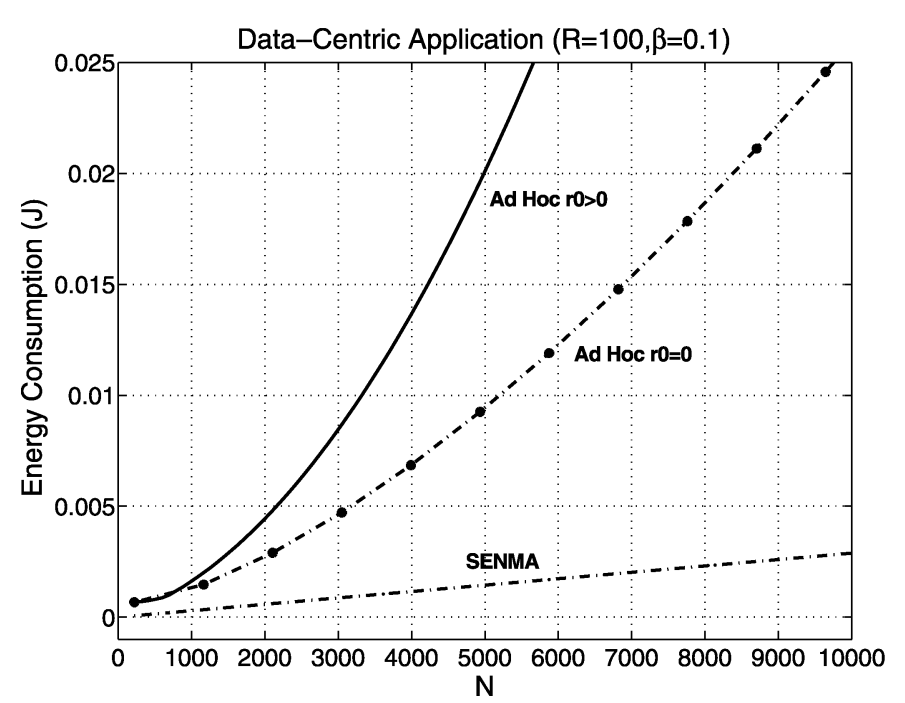

(a)

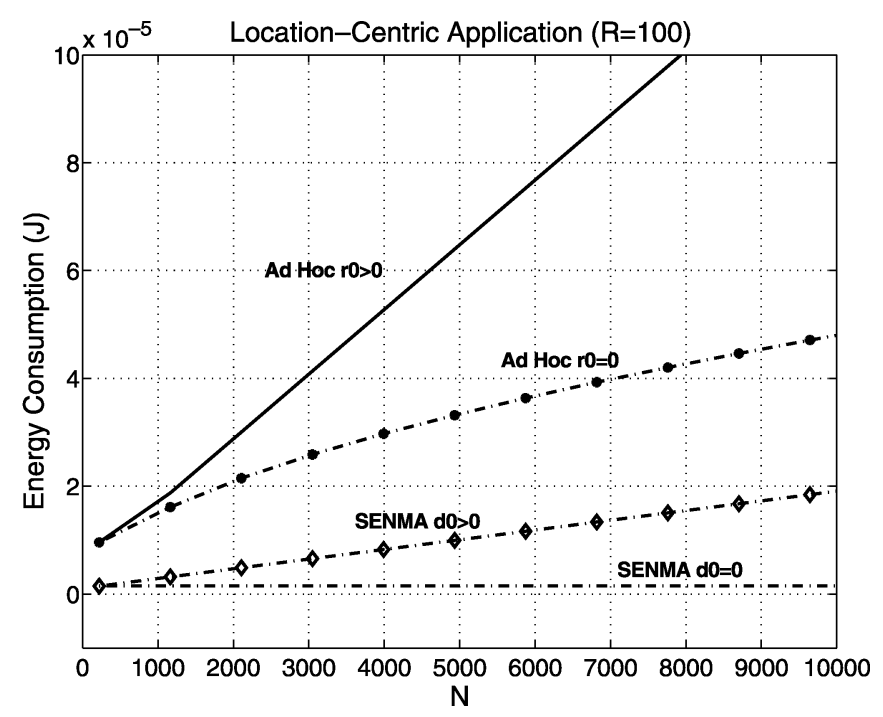

(b)

Fig. 8. Energy consumption of a query-reply operation. (a) Data-centric application. (b) Location-centric application. $(H=50 \mathrm{~m})$.

Shown in Fig. 8(a) are numerical results on the energy consumption. ${ }^{8}$ Here, we consider increasing the network size by increasing the density $\rho$. The shape of the curves confirms the scaling laws given in (30), (31). Orders of magnitude of improvement in energy efficiency can be achieved by SENMA in large sensor networks.

\section{Energy Efficiency for Location-Centric Applications}

In location-centric applications, the access point is interested in a particular geographic location in the sensor field. For the flat ad hoc architecture, the gateway node transmits the request to the area of interest using a predetermined route. One of the sensors located in the area of interest then transmits its measurement to the gateway node. Again, the calculation of the total energy consumption is similar to the point-to-point traffic analysis given in Section III

$$
\begin{aligned}
\mathcal{E}_{\mathrm{AdHoc}}^{(q)} & =2 \min _{r \geq r_{\min }}\left(E_{\mathrm{rx}}+E_{\mathrm{tx}}(r)+\frac{r^{2}}{R^{2}}(N-2) E_{\mathrm{ha}}\right) \frac{\mathbb{E}(X)}{r} \\
& = \begin{cases}\mathcal{O}(\sqrt{N \log N}), & \rho \uparrow, r_{0}=0 \\
\mathcal{O}(N), & \rho \uparrow, r_{0}>0 \\
\mathcal{O}\left(\sqrt{N(\log N)^{\alpha-1}}\right), & R \uparrow\end{cases}
\end{aligned}
$$

In SENMA, the mobile AP moves to the area of interest and activates sensors located in that area. Let $d_{0}$ denote the minimum radius of the coverage area of the mobile AP. The total energy consumption is given by

$\mathcal{E}_{\text {SENMA }}^{(q)}=\min _{d \geq d_{0}} \frac{d^{2}}{R^{2}} N E_{\mathrm{rx}}+E_{\mathrm{tx}}(H)= \begin{cases}\mathcal{O}(1), & \rho \uparrow, d_{0}=0 \\ \mathcal{O}(N), & \rho \uparrow, d_{0}>0 \\ \mathcal{O}(1), & R \uparrow\end{cases}$

Shown in Fig. 8(b) are numerical results where the network size is increased by increasing $\rho$. These results agree with the

\footnotetext{
${ }^{8}$ The values for the radio model are the same as used to obtain Fig. 2. Transmissions in a flat ad hoc sensor networks are between low-lying antennas, where signal decays as the fourth power of distance $(\alpha=4)$ due to partial cancellation by ground-reflected rays [29], [30]. In SENMA, we may have a free space between the sensor field and the mobile AP where $\alpha=2$.
}

scaling laws given by (32) and (33) and demonstrate the potential gain achieved by SENMA in large sensor networks.

\section{CONCLUSION}

We present in this paper an analytical approach to energy consumption of mobile ad hoc and large-scale sensor networks. Our goal is to investigate the interdependencies among network parameters and specify operation regions where reactive networking is more energy efficient than proactive networking. For instance, the analytical results obtained in this paper can be used to obtain the partition of the design space according to the energy efficiency of the proactive and reactive networking. Such a partition can only be described at the intuitive level previously [20]. The analysis presented in this paper allows the user to examine different design scenarios by setting specific network and channel parameters: the channel fading rate, the node mobility, the message arrival rates, etc. By examining the benefit of perfect wake-up scheme, our result also provides a quantitative assessment of the potential payoff of having a perfect schedule and precise clocks.

One should keep in mind that our analysis is not precise for specific scenarios, nor is it intended to match certain network implementation. Simplifying assumptions are made for they are necessary to make the analysis tractable. The results presented here should be viewed as a baseline benchmark with which simulation based approach can be cross-checked. Techniques used in the analysis may be applied to specific improvements on proactive and reactive routing such as route caching, local route repair, and probabilistic flooding, which is out of the scope of this paper.

As pointed out in [31] and [32], energy-aware networking can be addressed from two fundamentally different perspectives: energy efficient and energy limited operations. In the former case, it is implicitly assumed that unlimited energy resource is available; the goal is to find the most efficient way of using it. In the latter, each node has a hard constraint on the total energy. In this paper, we take the first perspective on energy-aware networking. 


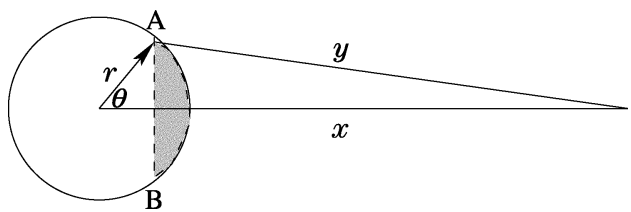

Fig. 9. Relaying zone from the source to the destination.

We point out that results obtained under one formulation does not directly apply to the other.

We have ignored energy consumption at the MAC layer by assuming a perfect MAC in which every transmission is successful. This idealistic assumption is for the purpose of obtaining an analytical benchmark and has been commonly used in, for example, the work of Gupta and Kumar [25] on the capacity of wireless network and other work on the performance analysis of routing protocols. It is our hope that the techniques presented in this paper will serve as a starting point in obtaining a more complete analytical framework for large-scale mobile ad hoc and sensor networks.

\section{APPENDIX A \\ PROOF OF PROPOSITION 1}

It is obvious that $h(x, r) \geq(\lceil x / r\rceil)$. To prove Proposition 1 , we will establish a probabilistic upper bound on $h(x, r)$ which is arbitrarily close to this lower bound.

We map the network to a disk with unit area. It is clear that the case of increasing $R$ is equivalent to the case of increasing $\rho$ without hardware limit $\left(r_{0}=0\right)$. We thus only consider the case of increasing $\rho$.

Shown in Fig. 9 is a source-destination pair with distance $x>r$. Consider the event when at least one node is located in the shaded area for relaying. This area is referred to as the relaying zone and is determined by $\theta$. Let $d_{\theta}(x, r)$ denote the distance covered in this hop. It is clear that the worst scenario (in terms of covered distance) occurs when the relaying node locates at $\mathrm{A}$ or $\mathrm{B}$. Thus

$$
\begin{aligned}
d_{\theta}(x, r) & \geq x-y \\
& =x-\sqrt{(x-r \cos \theta)^{2}+r^{2} \sin ^{2} \theta} \\
& =x-\sqrt{x^{2}+r^{2}-2 x r \cos \theta} \\
& >(1-\sqrt{2(1-\cos \theta)}) r
\end{aligned}
$$

where we have used the fact that $x-\sqrt{x^{2}+r^{2}-2 x r \cos \theta}$ increases with $x$ for $x>r$.

Assume that in each hop, the packet is relayed by one node in the relaying zone. The lower bound on $d_{\theta}(x, r)$ leads to an upper bound on $h(x, r)$ as

$$
h(x, r)<\left\lceil\frac{x}{(1-\sqrt{2(1-\cos \theta)}) r}\right\rceil .
$$

We now demonstrate that there exists $\theta_{0}>0$ (independent of $N)$ such that for any $0<\theta<\theta_{0}$, the probability $p_{\theta}(N, r)$ of having at least one empty relaying zone from the source to the

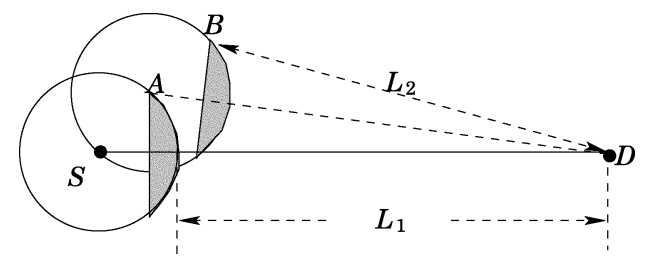

Fig. 10. Relaying zone from the source to the destination.

destination (thus, the upper bound given in (34) does not hold) approaches to 0 as $N \rightarrow \infty$.

Lemma 1: There exists $\theta_{0}>0$ independent of $N$ and $r$ such that all relaying zones associated with relaying nodes at least $2 r$ away from the destination do not overlap for any $0<\theta<\theta_{0}$.

Proof: As shown in Fig. $10, \mathcal{Z}_{1}(\theta)$ and $\mathcal{Z}_{2}(\theta)$ denote the first two relaying zones toward the destination $\mathrm{D}$ that are $x$ away from the source $\mathrm{S}$. We first show that $\mathcal{Z}_{1}(\theta)$ and $\mathcal{Z}_{2}(\theta)$ do not overlap for $\theta$ sufficiently small.

Let $L_{1}$ denote the minimum distance from a point in $\mathcal{Z}_{1}(\theta)$ to the destination, and $L_{2}$ the maximum distance from a point in $\mathcal{Z}_{2}(\theta)$ to the destination. To show that $\mathcal{Z}_{1}(\theta)$ and $\mathcal{Z}_{2}(\theta)$ do not overlap, we only need $L_{1}>L_{2}$. Clearly, $L_{1}=x-r$ (see Fig. 10). We now compute $L_{2}$ as follows.

Let $x_{1}$ denote the remaining distance to the destination after the first hop. From Fig. 10, we see that $L_{2}$ is given by the distance from $B$ to the destination, i.e.,

$$
\begin{aligned}
L_{2}^{2} & =\left(x_{1}-r \cos \theta\right)^{2}+r^{2} \sin ^{2} \theta \\
& \leq\left(\sqrt{(x-r \cos \theta)^{2}+r^{2} \sin ^{2} \theta}-r \cos \theta\right)^{2}+r^{2} \sin ^{2} \theta
\end{aligned}
$$

where we have used the fact that

$$
x_{1} \leq \sqrt{(x-r \cos \theta)^{2}+r^{2} \sin ^{2} \theta} .
$$

The equality holds when the packet is relayed by a node at $A$ in the first hop (see Fig. 10). With simple computation, we have $L_{2} \leq x^{2}-4 x r \cos \theta+4 r^{2}$. It thus follows that:

$L_{2}^{2}-L_{1}^{2} \leq x^{2}-4 x r \cos \theta+4 r^{2}-(x-r)^{2}=3 r^{2}+2 x r-4 x r \cos \theta$

which is negative if $\cos \theta>((3 r+2 x) / 4 x)$. Hence, for $x>2 r$ and $\theta_{0}=\arccos (7 / 8), \mathcal{Z}_{1}(\theta)$ and $\mathcal{Z}_{2}(\theta)$ do not overlap for any $0<\theta<\theta_{0}$.

Let $L_{3}$ denote the maximum distance from a point in the third relaying zone $\mathcal{Z}_{3}(\theta)$ to the destination. We have $L_{1}>L_{2}>L_{3}$, i.e., there is no overlapping between the first and the third relaying zone. We then conclude that all relaying zones associated with relaying nodes at least $2 r$ away from the destination do not overlap.

Lemma 1 shows that for all $\theta$ sufficiently small, the relaying zones do not overlap when the packet is more than $2 r$ away from the destination. Substituting $x$ by $2 r$ in (34), we see that the number of hops that may involve overlapping relaying zones is upper-bounded by a constant $c_{0}=\lceil 2 /(1-\sqrt{2(1-\cos \theta)})\rceil$. We are now ready to derive $p_{\theta}(N, r)$ by considering, separately, the finite $\left(c_{0}\right)$ number of hops that may involve overlapping relaying zones and the rest possibly infinite number of hops carried by nonoverlapping relaying zones. 
Let $A_{\theta}(r)$ denote the area of the relaying zone. We have $A_{\theta}(r)=r^{2}(\theta-\cos \theta \sin \theta)$. It then follows that:

$$
\begin{aligned}
& p_{\theta}(N, r) \\
& \left\lceil\frac{x}{(1-\sqrt{2(1-\cos \theta)}) r}\right\rceil \\
& \left.=\quad \sum_{i=1} \mathrm{P}_{r} \text { there are } i \text { empty relaying zones }\right\} \\
& \leq \sum_{i=1}^{c_{0}} \mathrm{P}_{r}\left\{\text { there is no node in an area of } A_{\theta}(r)\right\} \\
& \left\lceil\frac{x}{(1-\sqrt{2(1-\cos \theta)}) r}\right\rceil \\
& +\sum_{i=c_{0}+1} \mathrm{P}_{r} \text { there is no node in an area } \\
& \leq c_{0}\left(1-A_{\theta}(r)\right)^{N}+\sum_{i=1}^{\text {of } \left.\left(i-c_{0}\right) A_{\theta}(r)\right\}}\left(1-i A_{\theta}(r)\right)^{N} \\
& \left\lceil\frac{x}{(1-\sqrt{2(1-\cos \theta)}) r}\right\rceil \\
& =c_{0}\left(1-A_{\theta}(r)\right)^{N}+\sum_{i=1} e^{N \log \left(1-i A_{\theta}(r)\right)} \\
& <c_{0}\left(1-A_{\theta}(r)\right)^{N}+\sum_{i=1}^{\left\lceil\frac{x}{(1-\sqrt{2(1-\cos \theta)}) r}\right.} e^{-N i A_{\theta}(r)} \\
& < \begin{cases}c_{0}\left(1-\frac{c_{1} \log N}{N}\right)^{N}+\frac{e^{-c_{1} \log N}}{1-e^{-c_{1} \log N}}, & \text { if } r_{0}=0 \\
c_{0}\left(1-c_{2}\right)^{N}+\frac{e^{-c_{2} N}}{1-e^{-c_{2} N}}, & \text { if } r_{0}>0\end{cases} \\
& \stackrel{N \rightarrow \infty}{\rightarrow} 0
\end{aligned}
$$

where $c_{1}>0,0<c_{2}<1$ are constants independent of $N$. We have used Lemma 1 to obtain (36). In (37), we have used $\log (1-x)<-x$ for $|x|<1$ and the fact that the total area of empty relaying zones $i A_{\theta}(r)$ is smaller than the network area 1 . In (38), we have used the connectivity condition $\pi r(N)^{2}=\log N / N$. Thus, with probability approaching 1 as $N \rightarrow \infty$, there is at least one node in each relaying zone to relay the packet. The upper bound on $h(x, r)$ given in (34) holds with probability approaching 1 . Since we can choose any $\theta<\theta_{0}$, this upper bound is arbitrarily close to the lower bound, which completes the proof of Proposition 1.

\section{ACKNOWLEDGMENT}

The authors would like to thank the anonymous reviewers for their invaluable suggestions and comments.

\section{REFERENCES}

[1] Chip-Scale Atomic Clock, DARPA-MTO. [Online]. Available: http://www.darpa.mil/mto/csac/

[2] M. Llyas, The Handbook of Ad Hoc Wireless Networks. Boca Raton, FL: CRC, 2002

[3] V. Rodoplu and T. H. Ming, "Minimum energy mobile wireless networks," IEEE J. Sel. Areas Commun., vol. 17, no. 8, pp. 1333-1344, Aug. 1999.
[4] J. Chang and L. Tassiulas, "Energy conserving routing in wireless ad hoc networks," in Proc. IEEE INFOCOM, 2000, pp. 22-31.

[5] J. Gomez, A. Campbell, M. Naghshineh, and C. Bisdikian, "Conserving transmission power in wireless ad hoc networks," in Proc. 9th Int. Conf. Network Protocols, Riverside, CA, Nov. 2001, pp. 24-34.

[6] Y. Xu, J. Heidemann, and D. Estrin, "Geography-informed energy conservation for ad hoc routing," presented at the ACM/IEEE Int. Conf. Mobile Comput. Netwo. (ACM MobiCom), Rome, Italy, Jul. 2001

[7] A. Misra and S. Banerjee, "MRPC: Maximizing network lifetime for reliable routing in wireless environments," in Proc. IEEE Wireless Commun. Netw. Conf. (WCNC), Orlando, FL, Mar. 2002, pp. 800-806.

[8] V. Shurbanov and J. Redi, "Energy-efficient flodding in mobile ad hoc networks," in Proc. 23rd Army Sci. Conf., 2002.

[9] V. Srinivasan, C. Chiasserini, P. Nuggehalli, and R. Rao, "Optimal rate allocation for energy-efficient multipath routing in wireless ad hoc networks," IEEE Trans. Wireless Commun., vol. 3, no. 13, pp. 891-899, May 2004.

[10] J. C. Cano and P. Manzoni, "A performance comparison of energy consumption for mobile ad hoc networks routing protocols," presented at the 8th Int. Symp. Modeling, Anal. Simulation Comput. Telecommun. Syst., San Francisco, CA, Aug. 2000.

[11] L. M. Feeney, "An energy consumption model for performance analysis of routing protocols for mobile ad hoc networks," Mobile Netw. Ap plicat., vol. 6, pp. 239-249, 2001.

[12] L. Miller, "Distribution of link distances in a wireless network," J. Res. Nat. Inst. Standards Technol., vol. 106, pp. 401-412, Mar--Apr. 2001.

[13] A. McDonald and T. Znati, "A path availability model for wireless ad hoc networks," in Proc. IEEE Wireless Commun. Netw. Conf. (WCNC), 1999, pp. 21-24.

[14] D. Turgut, S. Das, and M. Chatterjee, "Longevity of routes in mobile ad hoc networks," in Proc. IEEE Veh. Technol. Conf. (VTC), 2001, pp. 2833-2837.

[15] J. Gao. (2002, Aug.) Analysis of energy consumption for ad hoc wireless sensor networks using a bit-meter-per-Joule metric. IPN Progress Rep. 42-150, Tech. Rep. [Online]. Available: http://ipnpr.jpl.nasa.gov/ progress_report/42-150/150L.pdf

[16] M. Bhardwaj and A. Chandrakasan, "Bounding the lifetime of sensor networks via optimal role assignments," in Proc. INFOCOM, New York, Jun. 2002, pp. 1587-1596.

[17] S. Coleri, M. Ergen, and T. Koo, "Lifetime analysis of a sensor network with hybrid automata modeling," presented at the Proc. WSNA, Atlanta, GA, Sep. 2002.

[18] H. Zhang and J. Hou, "On deriving the upper bound of lifetime for large sensor networks," in Proc. MobiHoc, 2004.

[19] A. Safwat, H. S. Hassanein, and H. T. Mouftah, "Structured proactive and reactive routing for wireless mobile ad hoc networks," in The Handbook of Ad Hoc Wireless Networks, M. Llyas, Ed. Boca Raton, FL: CRC, 2002.

[20] P. Samar, M. R. Pearlman, and Z. J. Haas, "Hybrid routing: The pursuit of an adaptable and scalable routing framework for ad hoc networks," in The Handbook of Ad Hoc Wireless Networks, M. Llyas, Ed. Boca Raton, FL: CRC, 2002.

[21] S. Giordano and I. Stojmenovic, "Position-based ad hoc routes in ad hoc networks," in The Handbook of Ad Hoc Wireless Networks, M. Llyas, Ed. Boca Raton, FL: CRC, 2002.

[22] C. Chiasserini and R. Rao, "Combining paging with dynamic power management," in Proc. IEEE INFOCOM, 2001, pp. 996-1004.

[23] J. Sheu, C. Hu, and C. Chao, "Energy-conserving grid routing protocol in mobile ad hoc networks," in The Handbook of Ad Hoc Wireless Networks. Boca Raton, FL: CRC, 2001.

[24] E. Shih, S. Cho, N. Ickes, R. Min, A. Sinha, A. Wang, and A. Chandrakasan, "Physical layer driven protocol and algorithm design for energy-efficient wireless sensor networks," in Proc. ACM MobiCom Rome, Italy, Jul. 2001, pp. 272-286.

[25] P. Gupta and P. R. Kumar, "The capacity of wireless networks," IEEE Trans. Inf. Theory, vol. 46, no. 2, pp. 388-404, Mar. 2000.

[26] P. Nicopolitidis, M. Obaidat, G. Papadimitriou, and A. Pomportsis, Wireless Networks. New York: Wiley, 2003.

[27] L. Tong, Q. Zhao, and S. Adireddy, "Sensor networks with mobile agents," in Proc. 2003 Int. Symp. Military Commun., Boston, MA, Oct. 2003, pp. 688-693.

[28] G. Mergen, Q. Zhao, and L. Tong, "Sensor networks with mobile access: Energy and capacity considerations," IEEE Trans. Commun., Mar. 2004, submitted for publication.

[29] K. Sohrabi, B. Manriquez, and G. Pottie, "Near ground wideband channel measurement," in Proc. 49th Veh. Technol. Conf., Houston, TX, May 1999, pp. 571-574. 
[30] G. Pottie and W. Kaiser, "Wireless integrated network sensors," Commun. ACM, vol. 43, pp. 51-58, May 2000.

[31] J. Wiesselthier, G. Nguyen, and A. Ephremides, "Energy-aware wireless networking with directional antennas: The case of session-based broadcasting and multicasting," IEEE Trans. Mobile Comput., vol. 1, no. 3, pp. 176-191, 2002.

[32] $\_$, "Resource management in energy-limited band-limited, transceiver-limited wireless networks for session-based multicasting," Comput. Netw., vol. 39, pp. 113-131, 2002.

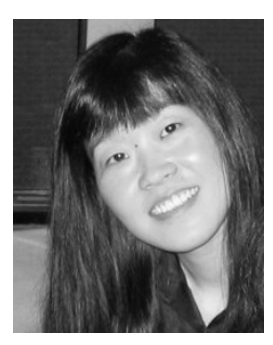

Qing Zhao (S'97-M'02) received the B.S. degree from Sichuan University, Chengdu, China, in 1994, the M.S. degree from Fudan University, Shanghai, China, in 1997, and the Ph.D. degree from Cornell University, Ithaca, NY, in 2001, all in electrical engineering.

From 2001 to 2003, she was a Communication System Engineer with Aware, Inc., Bedford, MA. She returned to academe in 2003 as a Postdoctoral Research Associate with the School of Electrical and Computer Engineering, Cornell University. In 2004, she joined the Department of Electrical and Computer Engineering, University of California, Davis, where she is currently an Assistant Professor. Her research interests are in the general area of signal processing, communication systems, wireless networking, and information theory. Specific topics include adaptive signal processing for communications, design and analysis of wireless and mobile networks, fundamental limits on the performance of large scale ad hoc and sensor networks, and energy constrained signal processing and networking techniques.

Dr. Zhao received the IEEE Signal Processing Society Young Author Best Paper Award.

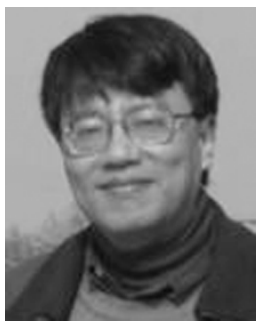

Lang Tong (S'87-M'91-SM'01-F'05) received the B.E. degree from Tsinghua University, Beijing, China, and the M.S. and Ph.D. degrees from the University of Notre Dame, Notre Dame, IN.

$\mathrm{He}$ is a Professor in the School of Electrical and Computer Engineering, Cornell University, Ithaca, NY. He was a Postdoctoral Research Affiliate at the Information Systems Laboratory, Stanford University, Stanford, CA. Prior to joining Cornell University, he was on the faculty at the University of Connecticut and the West Virginia University. He was also the 2001 Cor Wit Visiting Professor at the Delft University of Technology. His areas of interest include statistical signal processing, wireless communications, communication networks and sensor networks, and information theory.

Dr. Tong received the Young Investigator Award from the Office of Naval Research in 1996, and the Outstanding Young Author Award from the IEEE Circuits and Systems Society in 1994, the 2004 Best Paper Award from the IEEE Signal Processing Society, and the 2004 Leonard G. Abraham Prize from the IEEE Communications Society. 